\title{
Aplicabilidade do método modificado de Washburn para medidas de ângulo de contato de carbonato de cálcio
}

\section{(Applicability of the modified Washburn method to contact angle measurements of calcium carbonate)}

\author{
G. A. Valencia ${ }^{1}$, R. G. Pileggi ${ }^{1}$ \\ ${ }^{1}$ Universidade de São Paulo, Escola Politécnica, Departamento de Engenharia Civil, \\ Laboratório de Microestrutura e Ecoeficiência, São Paulo, SP, Brasil
}

\begin{abstract}
Resumo
Dispersões cerâmicas apresentam propriedades físicas e químicas fortemente influenciadas por forças superficiais entre partículas e o líquido. Como estratégia para diminuir impactos ambientais, dispersões cerâmicas estão tornando-se mais concentradas, resultando em sistemas mais complexos e mais susceptíveis às forças superficiais. Caracterizar a molhabilidade das partículas pelo líquido é uma forma de determinar o efeito dessas forças. Molhabilidade representa a grandeza de um líquido se espalhar sobre um sólido, descrita pelo ângulo de contato. Para pós, é recomendado o método modificado de Washburn, baseado na ascensão capilar do líquido em um tubo contendo o pó. Nesse trabalho objetivou-se medir valores de ângulo de contato de carbonato de cálcio em duas granulometrias com o propósito de avaliar a aplicabilidade do método modificado de Washburn para medidas de ângulo de contato de pós cerâmicos. As medidas de ângulo de contato utilizando aparato simplificado mostraram-se adequadas e sensíveis à granulometria, preparação da amostra e porosidade do sistema. Quanto menor a porosidade do sistema, menores foram os ângulos de contato calculados. Contudo, a variação dos valores calculados e da literatura sugere que os ângulos de contato calculados pelo método modificado de Washburn sejam comparativos, desde que se respeite a mesma configuração e preparação de amostra. Palavras-chave: molhabilidade, ângulo de contato, carbonato de cálcio, método modificado de Washburn.
\end{abstract}

\begin{abstract}
Dispersions of ceramics show physical and chemical properties strongly influenced by surface interactions between particles and liquid. As a strategy to reduce environmental impacts, ceramic dispersions are becoming more concentrated, resulting in complex systems and more susceptible to surface interactions. Characterizing the wettability of the particles by liquid is an effective way to determine the effect of surface interactions. Wettability is the tendency of a liquid to spread over a solid, described as contact angle. The modified Washburn method is recommended for powders, based on the capillary rise of the liquid into a packed bed of powder inside a tube. In this work, the objective is to measure the contact angle of calcium carbonate with two particle size distributions, for evaluating the applicability of the modified method of Washburn to contact angle measurements of ceramic powders. The contact angle measurements of calcium carbonate using simplified apparatus were adequate and sensitive to particle size distribution, sample preparation and porosity of the system. The lower the porosity of the system, the lower the calculated contact angles. However, the variation of calculated and literature values suggests that contact angles calculated by the modified Washburn method are comparative, once the same sample configuration and preparation are maintained.
\end{abstract}

Keywords: wettability, contact angle, calcium carbonate, modified Washburn method.

\section{INTRODUÇÃO}

Dispersões cerâmicas e materiais cimentícios no estado fresco podem ser considerados suspensões coloidais cujas propriedades reológicas são influenciadas por forças superficiais entre partículas e o líquido, na maioria dos casos água. No setor de materiais de construção civil, por exemplo, o concreto é o material de maior impacto ambiental devido ao seu elevado volume de produção, cerca de 10 bilhões de toneladas anuais, consumindo grandes quantidades de água e cimento, resultando em alta emissão de $\mathrm{CO}_{2}$ [1]. São possíveis estratégias para diminuição da emissão de $\mathrm{CO}_{2}$ a redução do uso de água e a substituição de parte do cimento por outros materiais compatíveis, chamados materiais cimentícios suplementares [1], como carbonatos de cálcio, escória, pozolanas e cinza volante, materiais que podem participar das reações de hidratação do cimento ou modificar a porosidade do sistema por efeitos físicos de pontos de nucleação e efeito filer [2-4]. Tais estratégias frequentemente tornam a suspensão mais concentrada e possivelmente mais susceptível às forças superficiais, tornando o sistema e seu comportamento reológico, físico e químico mais complexo. Dessa forma, o campo de estudo que visa compreender e prever o comportamento de suspensões cerâmicas em função de propriedades físico-químicas da superfície apresentase como campo de grande possibilidade e necessidade de exploração.

As principais forças atuantes na superfície de partículas 
imersas em meio aquoso são forças de van der Waals, forças eletrostáticas, movimento Browniano, forças de solvatação, hidrodinâmicas, capilaridade e forças estéricas [5]. Dessas forças, a força atrativa de van der Waals e repulsivas devido às forças eletrostáticas são amplamente exploradas através da teoria da estabilidade de coloides liofóbicos [6], também conhecido como teoria de DLVO, pela medida de potencial zeta [7-11]. Outra maneira igualmente efetiva de determinar o efeito das forças superficiais em suspensões, mas ainda pouco explorada, é estudar a molhabilidade e ângulo de contato das partículas pela água [12]. Molhabilidade é o fenômeno que representa a grandeza de um líquido se espalhar sobre um sólido e é fundamental na primária etapa de dispersão das partículas na água e na mistura. É um parâmetro físicoquímico, resultado do equilíbrio termodinâmico das fases sólido, líquido e vapor. As moléculas do líquido estão sujeitas a forças de coesão entre elas e adesão entre elas e outros materiais. O balanço dessas forças define o grau de molhabilidade de um líquido sobre determinado sólido. $\mathrm{O}$ indicador do grau de molhabilidade mais utilizado é o ângulo de contato $(\theta)$, definido pela equação de Young [13, 14], que representa o balanço de tensões superficiais $(\gamma)$ na interface entre as três fases sólido (S), líquido (L) e vapor (V), dado por:

$$
\cos \theta=\frac{\gamma_{S V}-\gamma_{S L}}{\gamma_{L V}}
$$

Ângulos menores que $90^{\circ}$ indicammolhagem espontânea do sólido, acima de $90^{\circ}$ o líquido não molha o sólido e ângulo nulo indica completa molhagem [15]. Desta forma, quanto menor o ângulo de contato, maior a molhabilidade. $\mathrm{O}$ ângulo de contato pode ser medido diretamente pelo formato de uma única gota sobre o substrato, conhecido como método da gota séssil. No caso de pós, a gota pode ser posta sobre o pó compactado, no entanto há algumas limitações como a possibilidade do líquido dissolver ou dispersar o pó causando instabilidade no formato da gota [14], a superfície resultante pode ser rugosa mascarando os resultados ou baixos ângulos de contato levam a rápidas penetrações de líquido prejudicando a medição $[16,17]$. Para pós e materiais porosos é recomendado o método dinâmico de ascensão capilar adotando o método modificado de Washburn [13-16, 18-22]. Nesse caso, acompanha-se a taxa no qual o líquido é absorvido pelo pó empacotado no interior de um cilindro sob condições controladas. Acompanhando-se a altura de molhagem ou massa absorvida, é possível calcular indiretamente o ângulo de contato.

Devido à complexidade do fenômeno de molhagem, influenciada não apenas pelas características físicoquímicas do líquido, mas também por outros parâmetros como a rugosidade, tamanho de partícula, heterogeneidade química da superfície e dissolução parcial do pó no líquido, cada par líquido/sólido deve ser estudado caso a caso [14]. Embora amplamente utilizado, o ângulo de contato calculado pelo método modificado de Washburn não é diretamente comparável a outras técnicas, visto que o líquido está em constante movimento através do pó, não havendo equilíbrio das fases [19]. A complexidade de metrologia desse método é intensificada pela necessidade do uso de um solvente adequado para cálculo da constante geométrica [23] e o fato do empacotamento, diâmetro ou porosidade afetarem diretamente o comportamento de ascensão do líquido em alguns casos [13,22] e em outros não [24, 25]. Tensiômetro é o equipamento comercial que permite realizar medidas de ângulo de contato por diversos métodos, incluindo o método de Washburn. Em países emergentes como o Brasil, tensiômetros de boa qualidade e que apresentam recursos como acompanhamento online do gráfico, de fácil operação e com microbalança precisa devem ser importados por empresas de países desenvolvidos como Estados Unidos e Alemanha, encarecendo o produto. A adaptação de uma balança convencional, resultando em um aparato simplificado e semelhante ao tensiômetro, pode custar de 10 a 15 vezes menos [15]. Assim sendo, nesse estudo pretende-se medir valores de ângulo de contato de carbonato de cálcio em duas granulometrias com o propósito de avaliar a aplicabilidade do método modificado de Washburn para medidas de ângulo de contato de pós cerâmicos. Conjuntamente, propõe-se avaliar um aparato simplificado e o procedimento de preparação para medidas de ângulo de contato de pós cerâmicos.

\section{Fundamentação teórica}

Ao se aproximar o líquido de um capilar, espontaneamente esse o embebe, sendo esse fluxo ascendente do líquido resultado das forças atrativas entre o líquido e a parede do capilar, conhecida como forças de adesão. Bell e Cameron [26] foram os primeiros a demonstrar experimentalmente a relação linear entre o quadrado da altura da molhagem do capilar (h) com o tempo (t), disposto horizontal ou verticalmente. No entanto foi Washburn [18] que descreveu a dinâmica do fluxo capilar derivada da lei de Poiseuille e expressando na forma da Eq. B, no qual é possível determinar o valor do ângulo de contato $(\theta)$, um parâmetro físico-químico da superfície:

$$
\mathrm{h}^{2}=\left(\frac{\gamma}{\eta} \frac{\cos \theta}{2}\right) \mathrm{rt}
$$

em que, $\eta$ é a viscosidade do líquido, $\gamma$ a tensão de superfície do líquido e $\theta$ é o ângulo de contato do líquido no capilar de raio r. Esse mesmo fenômeno ocorre ao se considerar os vazios entre as partículas de uma certa unidade de pó compactado sejam um conjunto de capilares tortuosos e, assim, estender o modelo. Considerando as dimensões do tubo que contém o pó e a porosidade, por manipulação matemática, chega-se à equação modificada de Washburn (Eq. C), respeitando as seguintes condições: 1) o líquido deve ser Newtoniano e incompressível, 2) o escoamento deve ser laminar e estacionário, 3) o líquido deve apresentar 
velocidade nula na parede, 4) não há pressão externa e 5) efeitos gravitacionais são desprezíveis [13, 16, 19, 22]:

$\mathrm{m}^{2}=\frac{\mathrm{r}_{\mathrm{eq}} \mathrm{A}^{3} \varepsilon^{2}}{2}\left(\frac{\rho^{2} \gamma \cos \theta}{\eta}\right) \mathrm{t}=\mathrm{C}\left(\frac{\rho^{2} \gamma \cos \theta}{\eta}\right) \mathrm{t}$

no qual, m é a massa de solvente que embebe o pó, o é a densidade do líquido, $\eta$ é a viscosidade do líquido e t é o tempo. C é a constante do material que engloba fatores geométricos do empacotamento, descrita por $\mathrm{r}_{\mathrm{eq}} \mathrm{A}^{2} \varepsilon^{2} / 2$, em que $r_{\text {eq }}$ é o raio equivalente dos vazios, A é a área transversal do tubo e $\varepsilon$ é a porosidade. Nota-se que pela equação que esse método é aplicável apenas para ângulos menores que $90^{\circ}$ [14], uma vez que o cosseno seria negativo para ângulos maiores que $90^{\circ}$. Experimentalmente o valor de $\mathrm{C}$ pode ser calculado utilizando um líquido que molha totalmente, ou seja, $\theta=0^{\circ}$ e $\cos 0^{\circ}=1$, desde que se garanta a mesma preparação do pó para as medidas seguintes com outros líquidos, ou seja, mesma preparação da amostra. Os líquidos de referência mais comuns para o cálculo de C são alcanos, como pentano, hexano e octano, embora muitos pesquisadores utilizem metanol, etanol e tolueno. Alguns desses líquidos podem ser tão voláteis ou apresentarem alta taxa de penetração que dificultam as medidas exatas de massa [16]. Há ainda outras formas ou cuidados para calcular a constante geométrica $\mathrm{C}$, como pré-saturar o pó empacotado com o líquido de referência antes da medida para permitir a formação de um filme duplo na superfície do pó a fim de reduzir a energia livre necessária para atingir o equilíbrio com o vapor, pode-se ainda realizar medidas de referência no ponto isoelétrico, pois a taxa de molhagem mostra-se maior, ou utilizar outros métodos para calcular a constante geométrica $\mathrm{C}$ como porosimetria de intrusão de mercúrio [16].

Experimentos de ascensão capilar pelo método de Washburn usualmente resultam em curvas compostas de 3 etapas, conforme Fig. 1 [16]. A etapa I é rápida, as forças inerciais são dominantes e apresenta relação m vs. t linear, porém o recente encontro da extremidade do tubo pode perturbar as medidas. A etapa II é o regime de Washburn, governada pela força capilar, os vazios entre as partículas são preenchidos pelo líquido e a curva $\mathrm{m}^{2}$ vs. $\mathrm{t}$ é linear. $\mathrm{Na}$ etapa III, os vazios apresentam-se quase em sua totalidade preenchidos, cessando o ganho de massa, ou os poros das partículas passam a serem preenchidos.

A comparação direta de taxas de ganho de massa ${ }^{2}$, constante $\mathrm{C}$, cosseno e ângulo de contato permite inferências sobre a molhabilidade de diferentes líquidos para um mesmo pó ou do mesmo líquido para diferentes pós. Embora o método de Washburn seja muito utilizado, diferentes autores apresentam diversos resultados de valores de ângulo para sólidos semelhantes ou sobre o efeito da porosidade e tamanho de partícula [13, 20,24]. Tais diferenças podem ser atribuídas a duas dificuldades: a correta seleção do líquido de referência para cálculo da constante geométrica e o empacotamento do pó, pois o método requer a preparação de amostras idênticas para medidas com líquido de referência

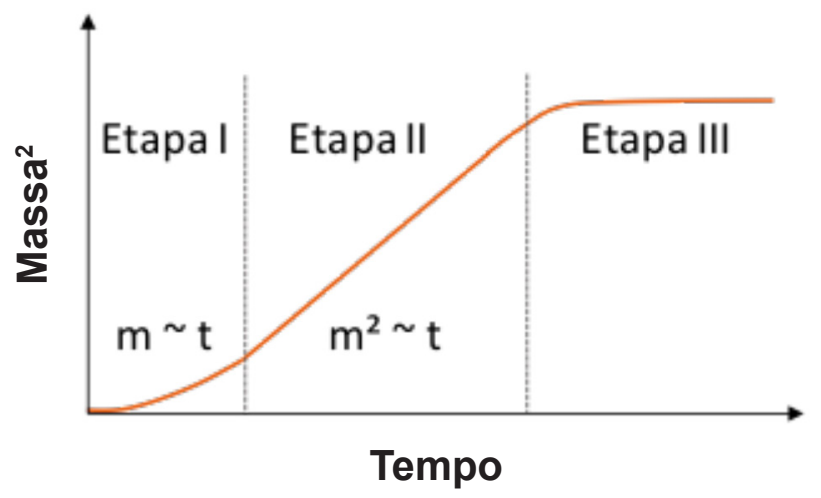

Figura 1: Perfil típico de massa ${ }^{2}$ por tempo de experimento pelo método modificado de Washburn, composto por três etapas distintas: etapa I é dominada pelas forças inerciais e apresenta massa linearmente proporcional ao tempo; etapa II é o regime de Washburn, onde forças capilares são dominantes e o quadrado da massa é linearmente proporcional ao tempo; na etapa III vazios estão quase em sua totalidade preenchidos, cessando o ganho de massa, ou ocorre o preenchimento dos poros das partículas.

[Figure 1: Typical mass ${ }^{2}$ vs. time profile of a modified Washburn experiment, composed of three distinct stages: stage 1 is dominated by inertial forces showing a linear $m$ vs. trelationship; stage 2 is the Washburn regime, where capillary forces are dominant showing a linear $m^{2}$ vs. $t$ relationship; in step III voids are fully filled, ceasing the mass gain, or the pores of the particles are being filled.]

e o líquido de estudo. O empacotamento pode ser afetado por diversos fatores, como a morfologia das partículas, porosidade do sistema, distribuição granulométrica, densidade das partículas e técnicas de compactação [6]. Amostras mal empacotadas levam a penetração irregular do líquido, diferente daquele apresentado na Fig. 1. Na literatura, não há uma técnica consolidada de preparação, tanto de inserção quanto compactação das partículas. Alguns autores optam por inserir todo o pó de uma única vez no tubo $[15,20,21]$ ou gradualmente $[27,28]$, seguido de repetidos golpes manuais ou mecânicos, de vibração [29], de vácuo [30] ou de centrifugação [13] até que certo empacotamento seja atingido. Dessa forma, a compactação das amostras é uma dificuldade em dois níveis. Primeiro durante o experimento, pela necessidade de dois empacotamentos idênticos, um para o líquido de referência e outro para o líquido de estudo. Segundo, ao comparar os valores calculados de ângulo com valores de literatura, pois não há unificação da preparação. Ainda assim, uma preparação de amostra que leve a um empacotamento uniforme e reprodutível é fundamental para obter resultados que respeitem o método modificado de Washburn e que possam ser comparados qualitativamente com a literatura.

\section{MATERIAIS E MÉTODOS}

Materiais: escolheu-se carbonato de cálcio natural micronizado da empresa Provale em duas granulometrias, Profine 1 e Procarb 5, provenientes da mesma jazida conforme fabricante. Ambos apresentaram massa específica de $2,74 \mathrm{~g} / \mathrm{cm}^{3}$ medida por picnometria a hélio 
(MultiPycnometer, Quantachrome Instruments). Para análise termogravimétrica considera-se a descarbonatação do carbonato de cálcio, que ocorre de 825 a $899^{\circ} \mathrm{C}$, conforme a reação:

$$
\mathrm{CaCO}_{3(\mathrm{~s})} \rightarrow \mathrm{CaO}_{(\mathrm{s})}+\mathrm{CO}_{2(\mathrm{~g})}
$$

Sabido que as massas molares dos compostos $\mathrm{CaCO}_{3}$, $\mathrm{CaO}$ e $\mathrm{CO}_{2}$ são, respectivamente, $100,1,56,1$ e $44 \mathrm{~g} / \mathrm{mol}$, a descarbonatação de $1 \mathrm{~mol}$ de carbonato de cálcio $\left(\mathrm{CaCO}_{3}\right)$, $44 \mathrm{~g}$ ou aproximadamente $44 \%$ de massa seria perdida. A Fig. 2 apresenta as curvas de perda de massa e da primeira derivada em relação ao tempo (DTG). Os calcários apresentaram perda de massa em apenas um momento, confirmado pela DTG, por volta de $830{ }^{\circ} \mathrm{C}$, correspondente à descarbonatação do carbonato de cálcio. O P1 apresentou perda de massa de $43,8 \%$ e massa residual de $56,2 \%$. Já o P5 obteve perda de massa de $41,4 \%$ e massa residual de $58,6 \%$. A Fig. 3 apresenta o resultado do ensaio de difração de raios $\mathrm{X}$ (DRX) para os dois carbonatos de cálcio, Profine $1 \mathrm{e}$ Procarb 5. Observou-se reposta semelhante para os dois pós. Qualitativamente, foi possível identificar picos referentes à calcita e dolomita, sendo que o pico mais evidente é o da calcita $\left(29^{\circ}\right)$. Pelos dados de termogravimetria e difração de raios X, pôde-se considerar Profine 1 e Procarb 5 majoritariamente carbonato de cálcio e semelhantes, confirmando a origem da mesma jazida. A distribuição granulométrica foi determinada por difração de raios laser (Helos/Sucell, Sympatec); Profine 1 e Procarb 5 apresentaram diâmetro médio de partícula de, respectivamente, 3 e $10 \mu \mathrm{m}$ e $90 \%$ do pó foram menores que, respectivamente, 8,48 e 31,4 $\mu \mathrm{m}$ (Fig. 4). Características da microestrutura das partículas foram analisadas por microscópio eletrônico de varredura (MEV) FEI Quanta 600 FEG, resolução 1024x884, imagens geradas por elétrons secundários e amostras preparadas por dispersão em ar. Visto na Fig. 5, o Profine 1 foi mais aglomerado, ambos apresentaram diversidade de tamanho de partículas, o formato foi anguloso e respeitaram os tamanhos apresentados na curva granulométrica. Utilizouse o solvente n-hexano P.A. $100 \%$ da Synth, densidade $0,66 \mathrm{~g} / \mathrm{cm}^{3}$ e viscosidade $45 \mathrm{cSt}$ conforme fabricante, para o cálculo da constante $\mathrm{C}$. As medidas de ângulo de contato foram feitas com água deionizada (densidade $0,997 \mathrm{~g} / \mathrm{cm}^{3} \mathrm{e}$ viscosidade $0,89 \mathrm{mPa} . \mathrm{s})$. Os experimentos foram executados à temperatura ambiente de $23{ }^{\circ} \mathrm{C}$. A tensão superficial dos líquidos foi medida pelo método da gota pendente utilizandose goniômetro DSA25E da Kruss e software DSA1.

Descrição do aparato simplificado: as medidas de ascensão capilar para utilização do método de Washburn foram realizadas em um aparato simplificado semelhante ao de outros autores [15, 23], apresentado na Fig. 6a, sendo (1) balança de precisão (Ohaus, Adventurer ARA520, resolução de $10 \mathrm{mg}$ ), (2) suporte de madeira com furo no centro, (3) tripé metálico de laboratório, (4) tubo de vidro que contém a amostra e (5) béquer com o líquido sobre a mesa de elevação manual. Antes das medidas, o pó permaneceu a $50{ }^{\circ} \mathrm{C}$ por pelo menos $12 \mathrm{~h}$. Após preparação, o tubo de vidro contendo

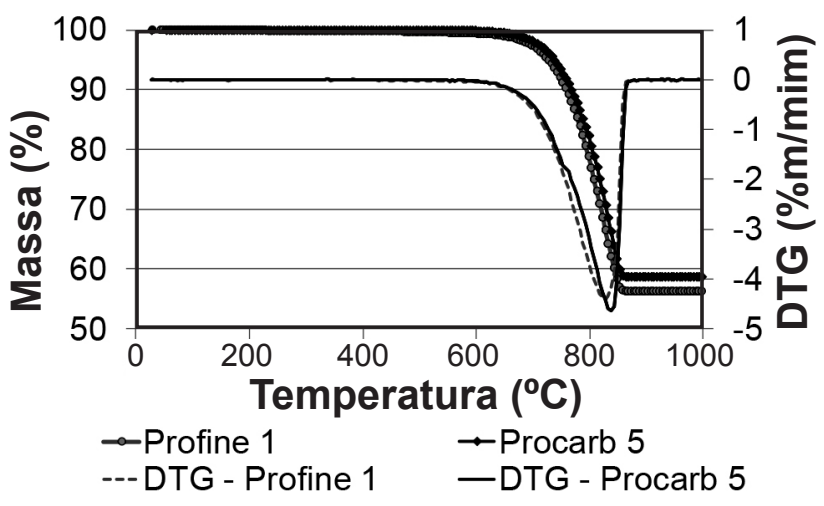

Figura 2: Curva de perda de massa e a primeira derivada (DTG) para os carbonatos de cálcio: Profine 1 e Procarb 5.

[Figure 2: Mass loss curve and first derivative (DTG) for the calcium carbonates: Profine 1 and Procarb 5.]

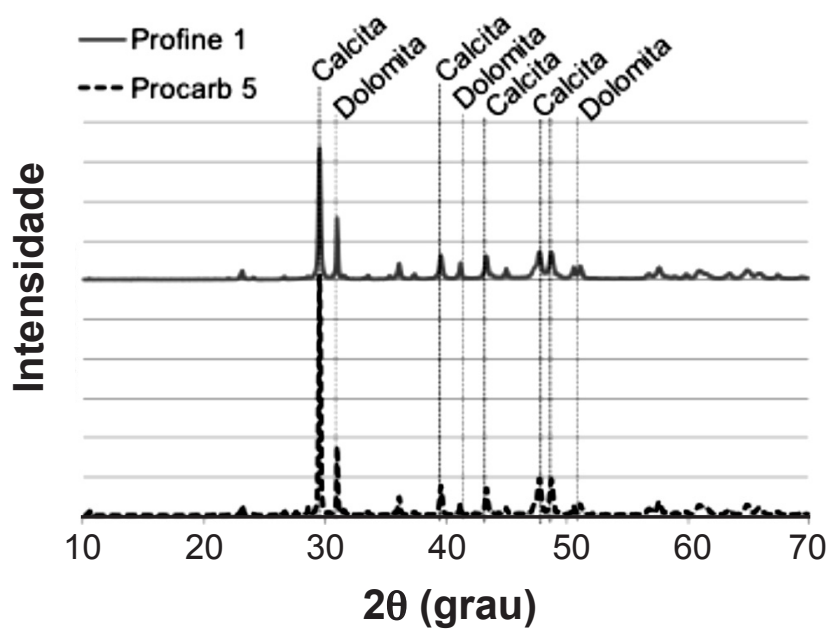

Figura 3: Difratogramas de raios $\mathrm{X}$ para os carbonatos de cálcio: Profine 1 e Procarb 5.

[Figure 3: X-ray diffraction patterns for the calcium carbonates Profine 1 and Procarb 5.]

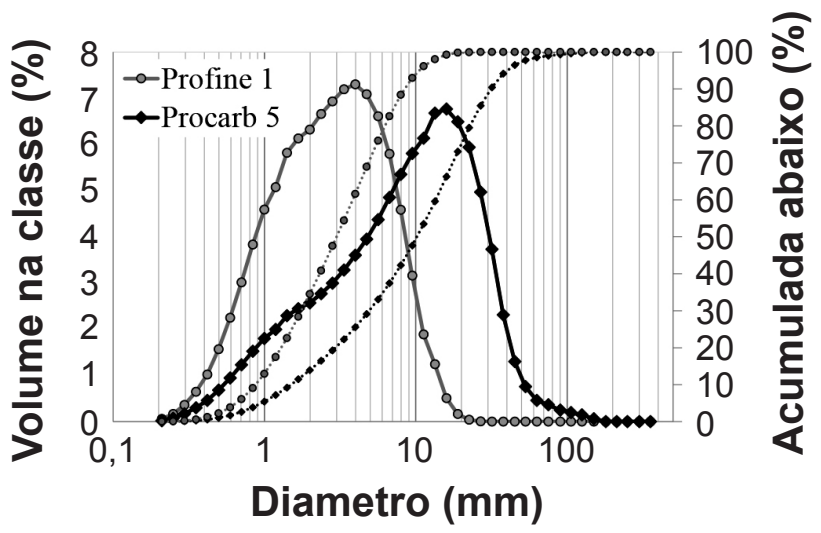

Figura 4: Distribuições granulométricas discreta e acumulada do Profine 1 e Procarb 5.

[Figure 4: Discrete and cumulative particle size distributions of Profine 1 and Procarb 5.] 


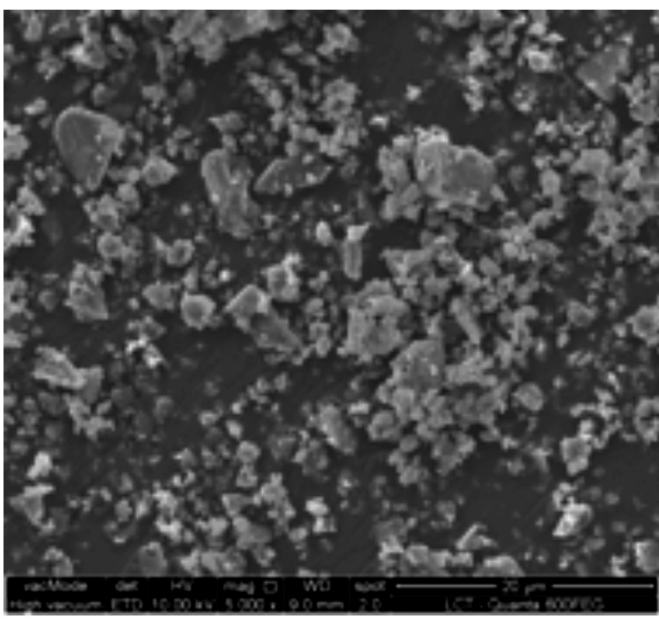

a)

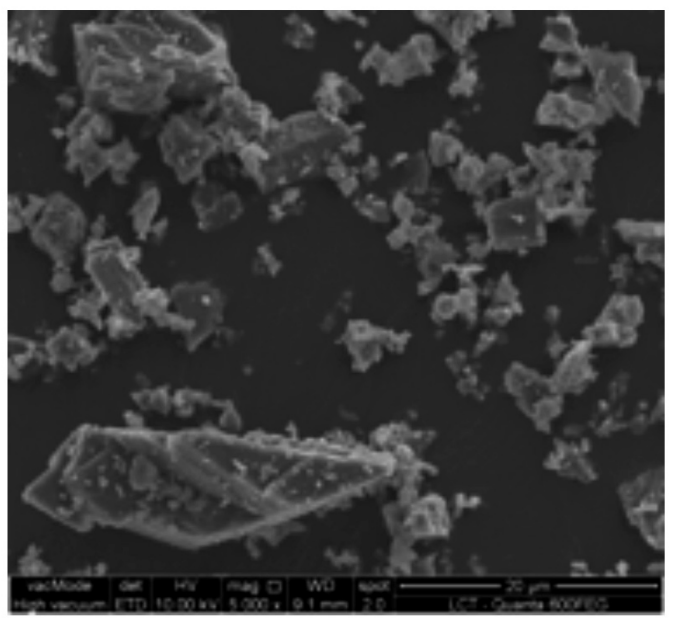

b)

Figura 5: Micrografias obtidas em microscópio eletrônico de varredura do Profine 1 e Procarb 5 (distância de trabalho, WD, de $\sim 9,0 \mathrm{~mm}$ ).

[Figure 5: Scanning electron micrographs of Profine 1 and Procarb 5 (working distance, $W D$, of $\sim 9.0 \mathrm{~mm}$ ).]

1,5 $\mathrm{g}$ da amostra foi preso por uma linha sob a balança e então foi zerada. O frasco contendo o líquido sob a mesa foi cuidadosamente elevado até que a superfície do líquido encostasse na extremidade do tubo. As medidas de ganho de massa em função do tempo foram coletadas a cada $30 \mathrm{~s}$ para uma planilha do Excel via software de coleta de dados (WinWedge, TALtech). A mesa foi vagarosamente abaixada após a massa permanecer praticamente constante (etapa III). O tubo de vidro utilizado apresentou diâmetro interno $10 \mathrm{~mm}$ e altura de $50 \mathrm{~mm}$, anel de fixação em aço inox e O-ring, da Kruss, SH0810 (Fig. 6b). É comum a escolha por tubos de vidro por serem química e fisicamente resistentes e transparentes [16]. Ser quimicamente resistente permite testar diferentes solventes. O vidro suporta a aplicação de diferentes esforços físicos e mecânicos para compactação, como golpes, vibração, vácuo ou centrifugação. A transparência auxilia na visualização do empacotamento e da penetração do líquido. Utilizou-se papel filtro como suporte na extremidade inferior do tubo sobre o anel de fixação, permitindo rápida passagem do líquido e sustentação do pó dentro do tubo, para que não caísse sobre o líquido. Durante o experimento, o papel filtro molha antes que o pó, assim o conjunto formado pelo tubo e o suporte deve apresentar molhagem rápida, menos que $10 \mathrm{~s}$, de preferência de 1 a 2 s [16]. Verificou-se a interferência do conjunto tubo e papel filtro através de experimentos sem amostra. Verificou-se que o ganho de massa do papel filtro e tubo pode ser negligenciado ( $\sim 0,01 \mathrm{~g})$ e que o tempo de molhagem foi de $\sim 5 \mathrm{~s}$. Dessa forma, o suporte mostrou-se adequado, de rápida e baixa absorção. Os ensaios foram realizados a $23 \pm 2{ }^{\circ} \mathrm{C}$ e após cada ensaio o tubo foi lavado com água deionizada e seco com papel antes da próxima medida. Inicialmente, analisouse o gráfico de massa e massa ao quadrado em função do tempo a fim de verificar visualmente as etapas teóricas da curva de ascensão capilar (Fig. 1). Em seguida, para ensaios com o solvente n-hexano, calculou-se o coeficiente angular da porção linear (etapa II) de pelo menos $30 \mathrm{~s}$ excluindo intervalo inicial (etapa I) de pelo menos $5 \mathrm{~s}$. Com o valor do coeficiente angular foi possível calcular a constante $\mathrm{C}$ pela Eq. C, assumindo que o ângulo de contato é $0^{\circ}\left(\log 0 \cos 0^{\circ}=1\right)$ devido ao baixo valor de tensão superficial. Esse modo de cálculo e solvente são usualmente utilizados para calcular a constante do material [13, 15, 19], embora Susana et al. [23] mostraram que para pós minerais os alcanos podem não ser a melhor escolha. Após determinado o valor de $\mathrm{C}$ e o valor do coeficiente angular para as curvas de água, foi possível calcular o cosseno e valores do ângulo de contato, conforme Eq. C, e assim avaliar a molhabilidade do pó carbonato de cálcio pela água.

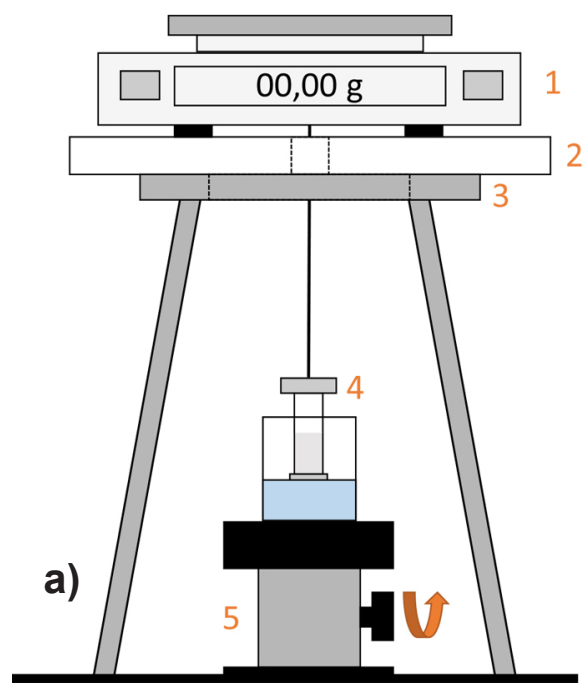

b)

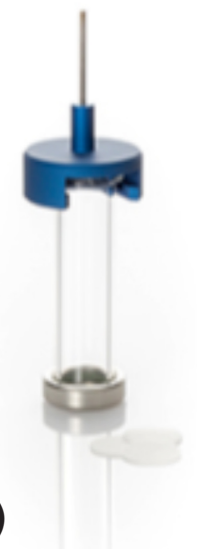

Figura 6: Aparato simplificado para medidas de ascensão capilar: (1) balança de precisão, (2) suporte de madeira com furo no centro, (3) tripé metálico de laboratório, (4) tudo de vidro e acessórios e (5) mesa de elevação manual por rosca.

[Figure 6: Simplified apparatus for Washburn capillary rise measurements: (1) analytical balance, (2) wooden support with center hole, (3) laboratory tripod, (4) glass tube and accessories, and (5) manual lifting table.]

Preparação das amostras: a adequação do aparato ao método modificado de Washburn para materiais cerâmicos 

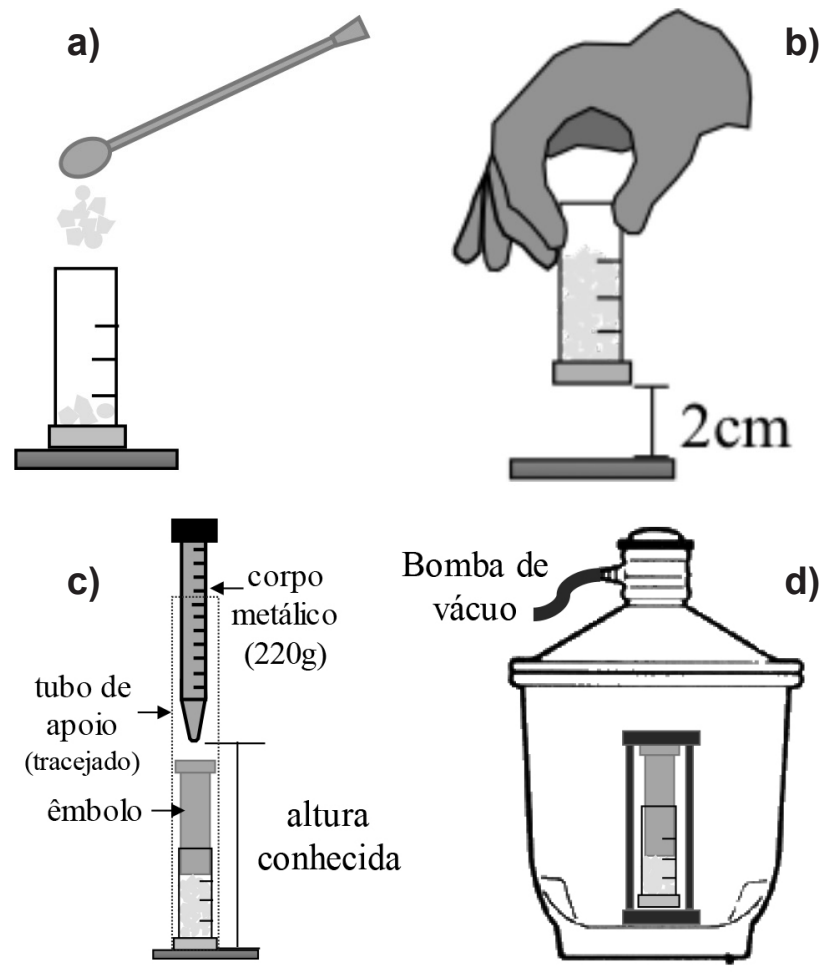

Figura 7: Ilustrações das formas de preparo: (a) preenchimento do tubo manualmente com auxílio de espátula; (b) impacto na mesa após abandono do tubo a altura de $2 \mathrm{~cm}$; (c) golpes com corpo metálico de $220 \mathrm{~g}$ sobre amostra a altura conhecida, uso de êmbolo plástico e tubo de apoio para alinhamento; e (d) vácuo por $2 \mathrm{~min}$ em dessecador, êmbolo fixado.

[Figure 7: Schematics of packing methods: (a) tube was filled manually with a spatula; (b) tube was vertically tapped against the table from a $2 \mathrm{~cm}$ height; (c) above the sample, plastic plunger was directly tapped with metallic body of $220 \mathrm{~g}$ from a known height, support tube for alignment; and (d) vacuum for 2 min in desiccator, fixed plunger.]

foi feita analisando a preparação da amostra de carbonato de cálcio Procarb 5. Foram testados 4 procedimentos de preparação das amostras de carbonato de cálcio no tubo de vidro, a fim de estabelecer uma combinação que seja reprodutível, de fácil e rápida preparação. Os procedimentos estudados foram (Fig. 7): a) preenchimento do tubo; b) impactos sobre mesa após abandono do tubo a altura de $2 \mathrm{~cm}$ (queda livre); c) impactos (4,6 ou 10) com massa conhecida a uma altura conhecida $(7,5,8,5$ ou 9,5 cm); e d) vácuo por $2 \mathrm{~min}$. O preenchimento (Fig. 7a) foi feito com espátula, seguida de impactos sobre mesa (Fig. 7b), ou de preenchimento em duas frações iguais de massa $(0,75 \mathrm{~g})$ combinado com impactos sobre mesa após cada metade. Com auxílio de um êmbolo de plástico e um corpo metálico de 220 g foi possível golpear verticalmente a amostra em diferentes alturas (Fig. 7c) utilizando um tubo externo para alinhamento. O tubo foi submetido ao vácuo por $2 \mathrm{~min}$ em dessecador (Fig. 7d). Evitou-se o deslocamento do pó no tubo fixando o êmbolo na altura final após os golpes e, em seguida, submetendo-o ao vácuo. Para amostra em certa combinação de preparação, foram feitas medidas ao menos em triplicata. Em poucos casos, quando duas curvas já ficavam muito próximas, não foi realizada a terceira medida.

\section{RESULTADOS E DISCUSSÃO}

Adequação do método modificado de Washburn para medidas de molhabilidade: para se empregar o método de Washburn, mediu-se a tensão superficial dos líquidos pelo método da gota pendente utilizando o método complexo de Young-Laplace [31]. Para água e n-hexano encontrou-se, respectivamente, os valores de 70,2 e $17,0 \mathrm{mN} / \mathrm{m}$ a $23{ }^{\circ} \mathrm{C}$, comparáveis a literatura, $71,99 \mathrm{mN} / \mathrm{m}$ para água a $25^{\circ} \mathrm{C}$ [32] e $18,4 \mathrm{mN} / \mathrm{m}$ para $\mathrm{n}$-hexano a $20^{\circ} \mathrm{C}$ [13]. A Fig. 8 mostra a diferença na forma da gota. $\mathrm{O}$ n-hexano molhou facilmente a agulha resultando em uma gota menor. Os valores medidos foram utilizados para os cálculos posteriores. A Fig. 9 exibe um exemplo de ensaio feito com o carbonato de cálcio e água no aparato apresentado na Fig. 6 que representa a maioria dos ensaios realizados. A linha tracejada corresponde à medida bruta de massa e a linha cheia o quadrado da mesma, ambas em função do tempo. Observou-se que o perfil medido foi conforme o esperado (Fig. 1), composta por três etapas. A etapa I apresentou duração de $5 \mathrm{~s}$ e maior coeficiente de correlação para a curva massa vs. tempo $\left(\mathrm{R}^{2}=0,9871\right.$ contra 0,9476). A máxima duração dessa primeira etapa para os demais ensaios foi de $\sim 7,5 \mathrm{~s}$, dentro do recomendado de até $10 \mathrm{~s}$. Na etapa II foi visível a maior linearidade da curva de massa ${ }^{2}\left(\mathrm{R}^{2}=0,9928\right.$ contra 0,9711$)$. Para os demais ensaios, essa etapa durou em média $33 \mathrm{~s}$ para o n-hexano e 64 s para água. Na etapa III, o ganho de massa foi muito pequeno. Dessa forma, pode-se afirmar que a configuração experimental desenvolvida resultou em perfis semelhantes apresentados por outros autores [13, 15, 16, 22]; logo, o método modificado de Washburn mostra-se adequado para medidas de ângulo de contato de materiais particulados cerâmicos.

Adequação da preparação das amostras de carbonato de cálcio: para aplicação do método de Washburn deve-se determinar uma preparação da amostra que garanta mesmo empacotamento para se obter medidas reprodutíveis. Aliada a essa necessidade, a adequação do aparato simplificado para amostras cerâmicas com balança menos precisa foi verificada através da combinação de quatro procedimentos de preparação. Para tal, utilizou-se apenas o carbonato de cálcio Procarb 5. Os quatros procedimentos testados foram detalhados anteriormente, sendo eles: a) preenchimento do tubo; b) impacto sobre a mesa após queda livre a $2 \mathrm{~cm}$ de altura; c) golpes (4, 6 ou 10) em êmbolo a certa altura conhecida $(7,5,8,5$ ou 9,5 cm); e d) vácuo por $2 \mathrm{~min}$. A Tabela I apresenta a série de 6 combinações testadas e a Fig. 10 apresenta os resultados de massa ${ }^{2}$ em função do tempo das mesmas; destaca-se que apenas a combinação 1 apresenta escala maior de tempo. Exceto para as curvas da primeira combinação (1), notou-se que as curvas apresentaram formato semelhante ao esperado (Fig. 1) e que a massa de água que embebe o pó foi maior que a massa de n-hexano. Embora com mesmo formato, foi visível que a sequência de 
combinações $(1-4,6,5)$ levou a curvas menos dispersas, a divisão das três etapas também foi mais clara e a ascensão dos líquidos foi mais rápida.

A constante geométrica $\mathrm{C}$ de cada combinação da série foi calculada a partir da média dos coeficientes angulares
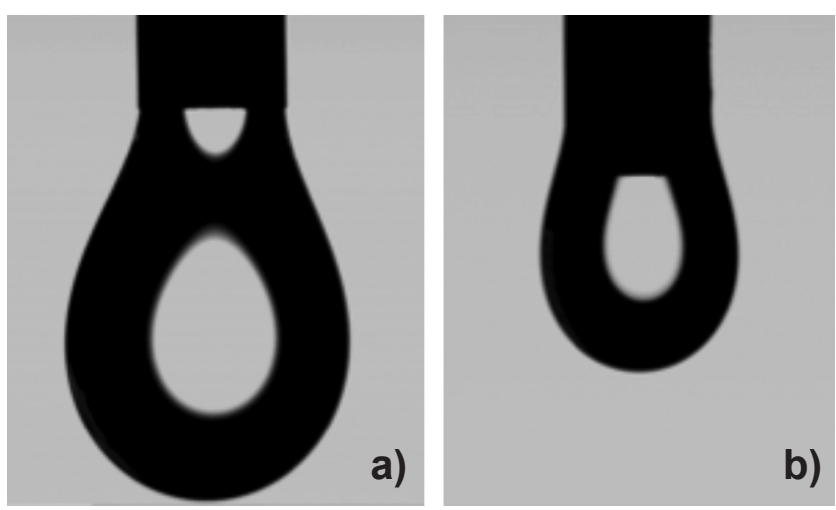

Figura 8: Imagens das gotas pendentes de água (a) e n-hexano (b) para o cálculo da tensão superficial.

[Figure 8: Images of water (a) and n-hexane (b) pedant drops for surface tension calculation.]

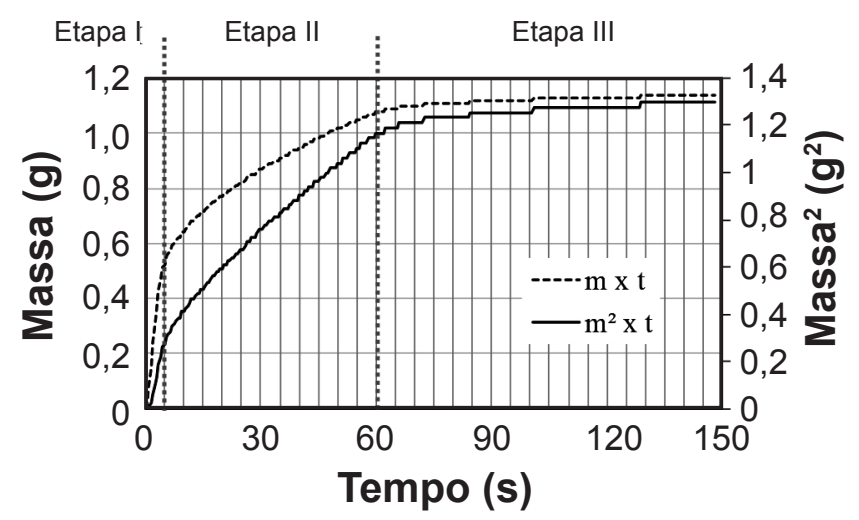

Figura 9: Exemplo de perfil dos ensaios realizados com a aparato experimental. Há três etapas, em conformidade com a literatura.

[Figure 9: Example of test profile performed on experimental apparatus. There are three stages, according to literature.] para medidas de n-hexano. Em seguida, com a média dos coeficientes angulares das curvas de água, calculou-se o cosseno e ângulo de contato de cada combinação seguindo a Eq. C. A Tabela II apresenta, para a série de combinações, os valores calculados da constante geométrica $\mathrm{C}$, taxa de penetração média das curvas de água com respectivo desvio-padrão $(\sigma)$ para combinações feitas pelo menos em triplicata, cosseno, valor do ângulo de contato (em grau) e a porosidade medida do sistema. A porosidade teórica do carbonato de cálcio Procarb 5 é 11,8\% calculado pelo modelo de Westman e Hugil [33], muito menor que aquelas medidas possivelmente pela dificuldade de preenchimento, empacotamento e repulsão eletrostática das partículas. A divergência observada na Fig. 10 para a combinação 1, no qual a água praticamente não molhou, pode ser consequência da porosidade do sistema $(64,9 \%)$, pois os vazios podem não formar canais com dimensões capilares, impedindo a ascensão de água, que ainda possui maior tensão de superfície e densidade que o n-hexano. $\mathrm{O}$ aparato simplificado mostrouse sensível à variação dos procedimentos de preparação do carbonato de cálcio estudado, levando a diferentes valores de ângulo de contato (Tabela II), apesar da balança ser pouco precisa e não totalmente dedicada. A combinação 5 mostrouse mais reprodutível, com menor desvio-padrão. Foi semelhante à combinação 6 , porém a 6 foi mais complexa por abranger a etapa de vácuo, demandando mais tempo e cuidado. Sendo assim, a combinação 5 mostrou-se mais reprodutível e simples para medidas de carbonato de cálcio. A série de combinações de preparação, especialmente a combinação 5, é provavelmente adequada para materiais cerâmicos no geral. Para essa combinação, o ângulo de contato do carbonato de cálcio Procarb 5 calculado foi de $42,7^{\circ}$. Os valores das constantes geométricas $\mathrm{C}$ calculadas com n-hexano, de 1,62 a $3,44 \times 10^{-16} \mathrm{~m}^{5}$ a $23{ }^{\circ} \mathrm{C}$, estão na mesma ordem de grandeza que valores da literatura de 4,60 a $54,2 \times 10^{-16} \mathrm{~m}^{5}$ calculadas com hexano a $20{ }^{\circ} \mathrm{C}$ [13] e 1,5 a $4,3 \times 10^{-16} \mathrm{~m}^{5}$ com octano à temperatura ambiente [22], embora diferem-se em uma ordem de grandeza do valor de $1,23 \times 10^{-15} \mathrm{~m}^{5}$ calculada com octano a $20{ }^{\circ} \mathrm{C}$ [20]. Tais variações podem depender tanto do líquido escolhido

Tabela I - Série de 6 combinações dos procedimentos de preparação do Procarb 5 para método de Washburn: a) preenchimento total ou parcial do tubo; b) impactos sobre a mesa do tubo após queda livre; c) golpes em êmbolo a altura conhecida; e d) vácuo por 2 min.

[Table I - Series of 6 packing methods combinations of Procarb 5 for Washburn capillary rise measurements: a) total or partial tube fill; b) tube tapped against the table from a $2 \mathrm{~cm}$ height; c) plastic plunger tapped from a known height; and d) vacuum for 2 min.]

\begin{tabular}{ccccc}
\hline Série & Preenchimento & Queda livre - tubo & Impacto em êmbolo & Vácuo \\
\hline 1 & Total & 40 impactos & - & - \\
2 & & - & 6 golpes a $8,5 \mathrm{~cm}$ & - \\
\hline 3 & & 4 golpes a $7,5 \mathrm{~cm}$ & - \\
4 & 2 partes & 10 impactos a cada & 6 golpes a $9,5 \mathrm{~cm}$ & - \\
5 & & metade & 10 golpes a $9,5 \mathrm{~cm}$ & - \\
6 & & 10 golpes a 9,5 cm & Sim \\
\hline
\end{tabular}


(1) total +40 impactos

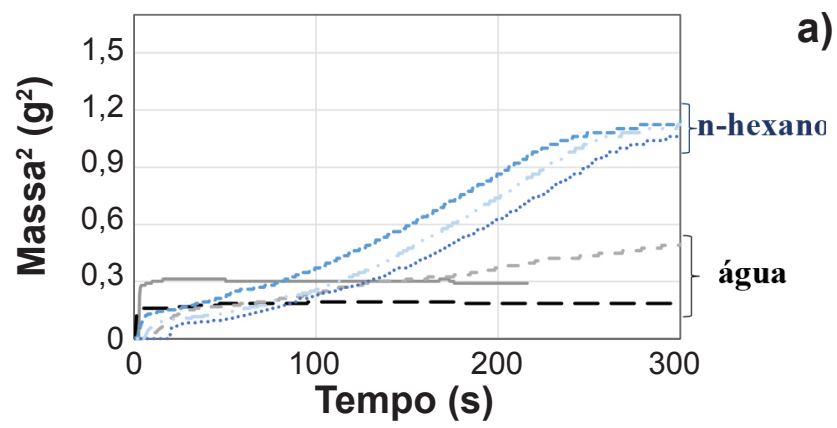

a)

\section{(3) 2 partes $+4 \times 7,5 \mathrm{~cm}$}

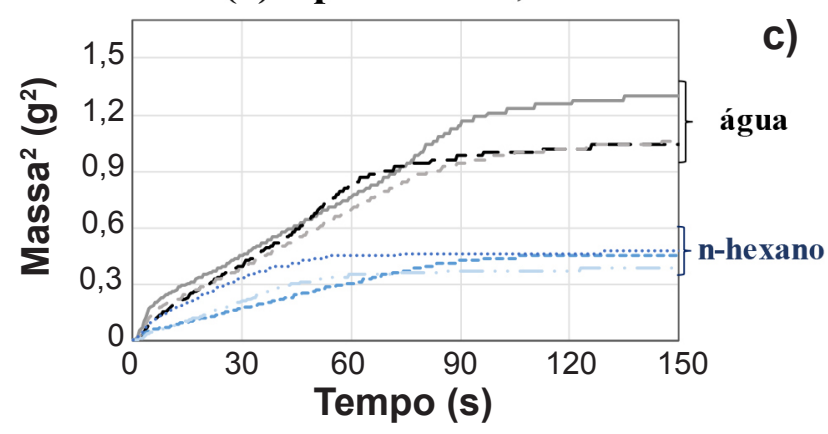

$\begin{array}{lll}\text { água } 1 & - \text {-água } 2 & -- \text { água } 3 \\ \text {----n-hexano } 1 & -\cdots \text { n-hexano } 2 & \text { ………n-hexano } 3\end{array}$ $\begin{array}{lll}\text { —água } 1 & - \text { água } 2 & -- \text { água } 3 \\ \text { …..... água } 4 & -\cdots \text { n-hexano } 1 \quad-\cdots \text { n-hexano } 2\end{array}$

b)

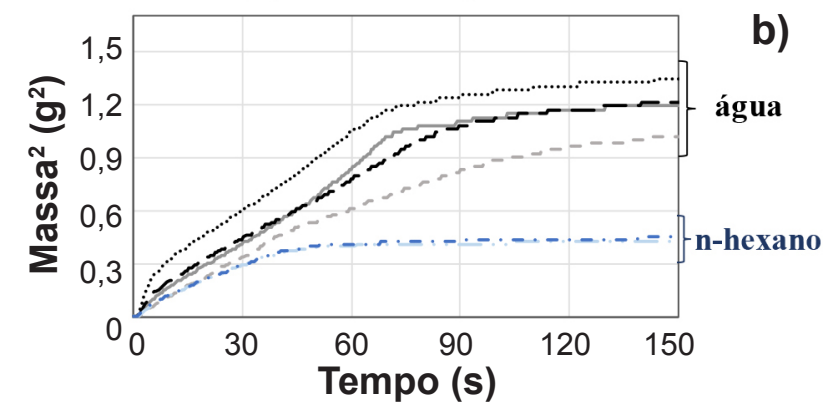

(2) total $+6 \times 8,5 \mathrm{~cm}$

$$
\text { água } 4
$$

\section{(4) 2 partes $+6 \times 8,5 \mathrm{~cm}$}

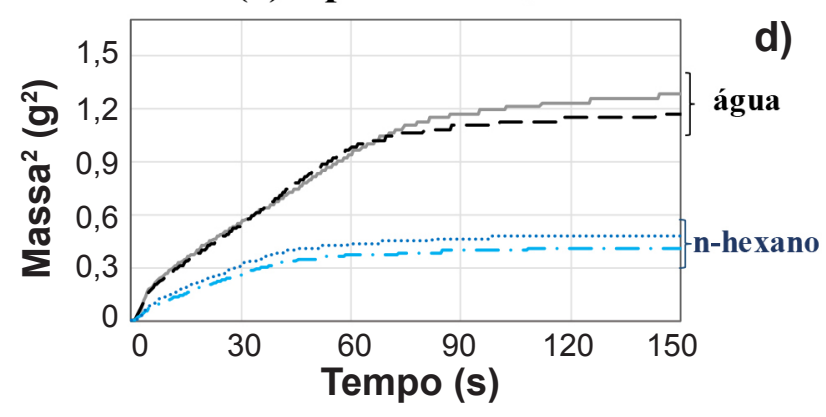

—água 1 - água $2-\cdots-n$-hexano $1 \cdots \cdots \cdots$ n-hexano 2

\section{(5) 2 partes $+10 \times 9,5 \mathrm{~cm}$}

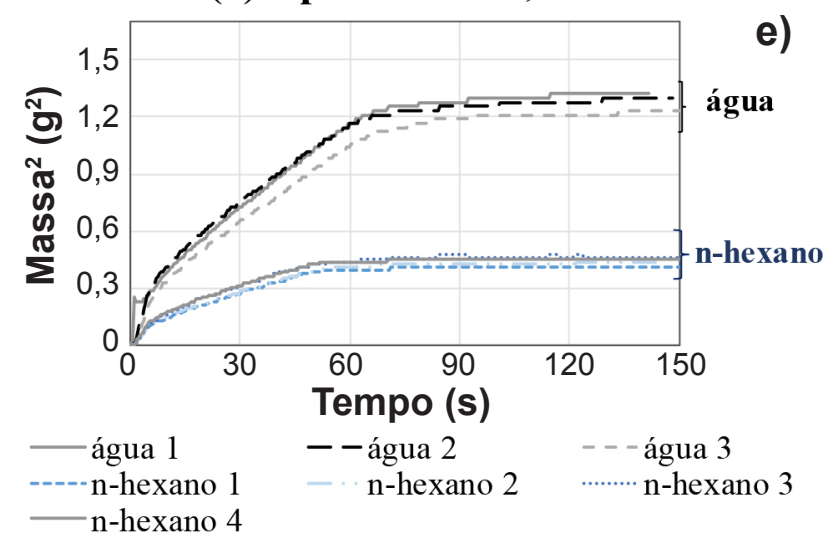

\section{(6) 2 partes $+10 \times 9,5 \mathrm{~cm}+$ vácuo}

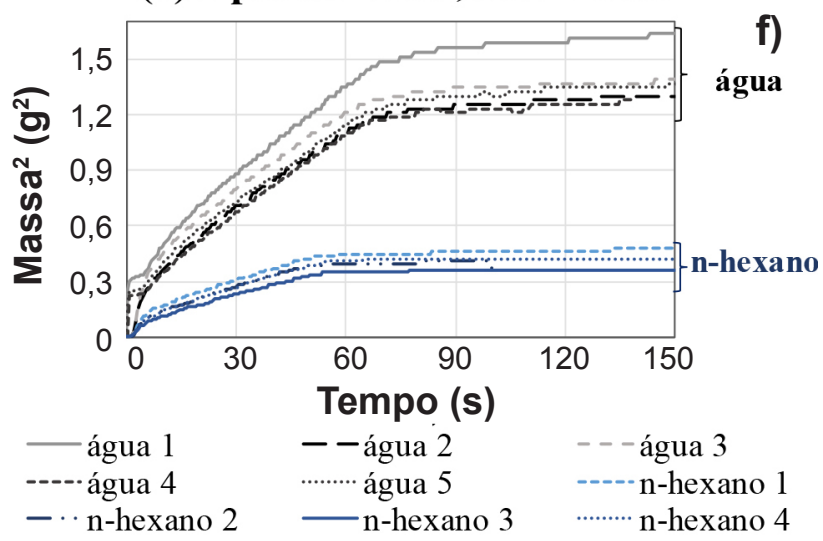

Figura 10: Curvas de massa² vs. tempo da série de 6 combinações de preparação de amostra de Procarb 5, para água e n-hexano.

[Figure 10: Mass $^{2}$ vs. time series of 6 packing methods combinations of Procarb 5, for water and n-hexane.]

e temperatura, pois diferenças das características físicoquímicas de tensão superficial, viscosidade e densidade afetam diretamente o cálculo de C (Eq. C), quanto do empacotamento do pó.

Efeito da granulometria e porosidade nas medidas de ângulo de contato: a Fig. 11 apresenta a relação entre o ângulo de contato em água e a porosidade do sistema para carbonatos de cálcio da série de combinações de preparação de amostras de Procarb 5 (Procarb 5 - série), para o carbonato de cálcio Profine 1 preparado segundo a combinação 5 e dados de autores que, do mesmo modo, analisaram carbonatos de cálcio pelo método de Washburn $[13,34]$. As linhas tracejadas correspondem aos valores de porosidades nominais do Procarb 5 e Profine 1, respectivamente, $11,8 \%$ e 15,6\%. É visto na Fig. 
Tabela II - Para cada combinação de preparação de amostra com Procarb 5: constante geométrica C média, taxa de penetração de água média, desvio padrão ( $\sigma$ ), cosseno, valor do ângulo de contato e porosidade.

[Table II - For each combination of sample packing method for Procarb 5: mean geometric constant C, mean water penetration rate, standard deviation $(\sigma)$, cosine, contact angle and porosity.]

\begin{tabular}{lccccccc}
\hline & \multirow{2}{*}{ Série } & n-hexano & \multicolumn{2}{c}{ Água } \\
& Cotal+40 pancadas & $\left.1,60^{16} \mathrm{~m}^{5}\right)$ & $\mathrm{m}^{2} / \mathrm{t}\left(\mathrm{g}^{2} / \mathrm{s}\right)$ & $\sigma\left(\mathrm{g}^{2} / \mathrm{s}\right)$ & $\cos \theta$ & $\theta\left({ }^{\circ}\right)$ & $\varepsilon(\%)$ \\
\hline 1 & Total+6x8,5 cm & 3,44 & 0,0012 & - & 0,09 & 84,7 & 64,9 \\
2 & 2 partes+4x7,5 cm & 2,69 & 0,0126 & 0,0016 & 0,45 & 63,0 & 48,3 \\
3 & 2 partes+6x9,5 cm & 3,19 & 0,0137 & - & 0,53 & 58,0 & 47,1 \\
4 & 2 partes+10x9,5 cm & 2,52 & 0,0150 & 0,0008 & 0,74 & 42,7 & 46,7 \\
5 & 2,48 & 0,0148 & 0,0010 & 0,74 & 42,3 & 46,6 \\
6 & 2 partes+10x9,5 cm+vácuo & 2,48 & 0,50 & 59,9 & 47,6 \\
\hline
\end{tabular}

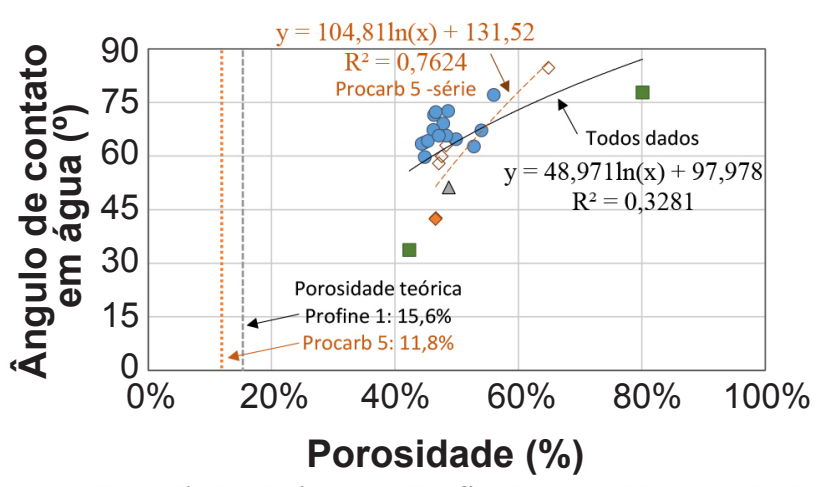

$\diamond$ Procarb 5 - Série $\Delta$ Profine $1 \quad \bullet[13] \quad \square[34]$

Figura 11: Ângulo de contato em água vs. porosidade de carbonatos de cálcio: série de combinações de preparo do Procarb 5, presente estudo (losangos abertos), destaque para a combinação 5 (losango fechado), Profine 1 na combinação 5 (triângulo), Galet et al. [13] (círculos), Depalo e Santomaso [34] (quadrados).

[Figure 11: Water contact angle vs. porosity of calcium carbonates samples: series of Procarb 5 packing methods combinations (open diamonds), combination 5 (closed diamond), Profine 1 in combination 5 (triangle), Galet et al. [13] (circles), Depalo and Santomaso [34] (squares).]

11 que os valores calculados de ângulo de contato em água para a série de combinações com o Procarb 5 e a medida de Profine 1 apresentaram molhagem espontânea do pó $\left(<90^{\circ}\right)$. Exceto a combinação 1 da série, os demais valores situaram-se dentro da faixa encontrada na literatura de $30<\theta<80^{\circ}[13,20$, 34]. No entanto, considerando todos os valores, desse estudo e da literatura, observa-se ampla variação desses valores para o que nominalmente é o mesmo material, carbonato de cálcio. Observa-se também que as porosidades medidas dos carbonatos de cálcio estudados são maiores que as teóricas.

Do presente estudo (losangos, Fig. 11), observa-se que o valor do ângulo de contato calculado diminuiu com a porosidade $\left(\mathrm{R}^{2}=0,76\right)$. Galet et al. [13] estudaram a preparação das amostras por centrifugação. Observaram que para amostras de talco, diâmetro médio de 19 um semelhante ao Procarb 5 , o ângulo de contato $\left(83^{\circ}\right.$ a $\left.88^{\circ}\right)$ também é proporcional à porosidade (66 a 71\%). Já carbonatos de cálcio (círculos na Fig. 11), diâmetros médios variando de 5 a $130 \mu \mathrm{m}$, não exibiram significativa relação entre ângulo de contato $\left(59,7\right.$ a $\left.77,1^{\circ}\right)$ e porosidade (44 a 56\%). Depalo e Santomaso [34] investigaram uma configuração experimental baseada no método modificado de Washburn, no qual acompanha-se o incremento de pressão. Dentre os materiais estão dois carbonatos de cálcio (quadrados na Fig. 11), diâmetros 6 e 528,8 $\mu$ m, que também apresentaram menor valor do ângulo de contato de equilíbrio (respectivamente $77,8^{\circ}$ e $33,7^{\circ}$ ) para o sistema com menor porosidade (respectivamente, $80,1 \%$ e $42,3 \%$ ). O Profine 1 , com menor diâmetro de partícula comparado com o Procarb 5 , apresentou maior ângulo de contato $\left(51,2^{\circ}\right)$ na mesma configuração de preparo (combinação 5), possivelmente devido à maior porosidade $(48,7 \%)$ que dificultou a penetração do líquido. Tal tendência foi observada em [22] com nylon, de 0 a $2000 \mu \mathrm{m}$, no qual maiores diâmetros resultaram em maiores vazios, menores taxas de penetração do líquido e menores valores de ângulo de contato. Kirchberg et al. [28] avaliaram a influência do formato e tamanho na molhabilidade de partículas magnéticas de ferro silício e de magnetita. Peneiraram os pós em frações que variaram o diâmetro médio de 12 a $87 \mu \mathrm{m}$ para ferro-silício e de 7 a $43 \mu \mathrm{m}$ para magnetita. Verificaram que o ângulo de contato decresce com aumento do diâmetro das partículas. Siebold et al. [20] investigaram a energia livre da superfície pela equação de Washburn. O carbonato de cálcio estudado apresenta partículas de 0 a $500 \mu \mathrm{m}$, no qual apenas $10 \%$ é menor que $100 \mu \mathrm{m}$, ou seja, maior que o Procarb 5. $\mathrm{O}$ ângulo de contato calculado foi maior $76,9^{\circ} \pm 4,4^{\circ}$. O maior ângulo de contato pode ser devido ao empacotamento menos eficiente e possível maior porosidade do sistema, uma vez que o método de preparação utilizado contempla apenas impactos do tubo sobre a mesa, semelhante à combinação (1).

Considerando todos os dados da Fig. 11, há fraca relação da porosidade de empacotamento com ovalor de ângulo $\left(R^{2}=0,33\right)$, sugerindo que as medidas de ascensão capilar pelo método de Washburn são influenciadas não apenas pela porosidade, mas possivelmente também pela distribuição dos poros e características físico-químicas das partículas. Possivelmente, medidas de ângulo de contato realizadas próximas da 
porosidade teórica poderiam representar diretamente as forças superficiais. A alta dispersão de valores de ângulo de contato em água dos carbonatos de cálcio estudados e da literatura sugere que os dados obtidos pelo método modificado de Washburn sejam predominantemente comparativos entre valores que respeitem mesma configuração e preparação da amostra. Ainda assim, esse método mostra-se importante e favorável a uma análise qualitativa para qualquer particulado cerâmico, por exemplo, se o líquido simplesmente molha o pó cerâmico (ângulo $<90^{\circ}$ ), como a mudança na forma, tamanho ou preparo do pó afeta a molhabilidade do sistema, qual líquido molha mais certo pó cerâmico ou se o mesmo líquido molha de forma similar pós cerâmicos diferentes. Observouse que o ângulo de contato possui relação proporcional à porosidade, visto nos ensaios em que a compactação durante a preparação das amostras foi alterada, pois a porosidade indica se os vazios do sistema formam canais capilares. De alguma forma, se o empacotamento do sistema for conhecido e os parâmetros que o afetam forem controlados e previstos, pode-se prever a molhabilidade do sistema para certo líquido, resultando em ganhos nos estudos reológicos. No método de Washburn, contudo, o empacotamento do material no tubo depende de muitos parâmetros que não foram completamente explorados, conhecidos e modelados, como morfologia, área superficial específica, distribuição granulométrica e densidade das partículas, afetados pela forma de compactação do sistema.

\section{CONCLUSÕES}

Medidas de ângulo de contato de carbonato de cálcio pelo método modificado de Washburn utilizando aparato simplificado foram adequadas por resultarem em perfis semelhantes ao teórico. A série de combinações de preparação sugerida no presente estudo, especialmente aquela elegida como mais reprodutível, é possivelmente satisfatória para outros materiais cerâmicos. Os ângulos de contato medidos para carbonatos de cálcio apresentaram-se sensíveis a granulometria e porosidade do sistema; quanto menor a porosidade do sistema, menor valor de ângulo de contato foi calculado. A variedade de valores de ângulo de contato do carbonato de cálcio sugere que os dados obtidos pelo método modificado de Washburn para qualquer material sejam comparativos entre valores que respeitam mesma configuração e preparação experimental.

\section{REFERÊNCIAS}

[1] C. Meyer, Cem. Concr. Compos. 31 (2009) 601.

[2] A.A. Ramezanianpour, E. Ghiasvand, I. Nickseresht, M. Mahdikhani, F. Moodi, Cem. Concr. Compos. 31 (2009) 715.

[3] B.L. Damineli, V.M. John, B. Lagerblad, R.G. Pileggi, Cem. Concr. Res. 84 (2016) 8.

[4] G. Bassioni, Appl. Energy 93 (2012) 132.

[5] E. Koos, N. Willenbacher, Science 331 (2011) 897.

[6] I.R. Oliveira, A.R. Studart, R.G. Pileggi, V.C. Pandolfelli,
Dispersão e empacotamento de partículas: princípios $e$ aplicações em processamento cerâmico, $2^{\mathrm{a}}$ Ed., Fazendo Arte Editorial, S. Paulo (2000).

[7] J. Plank, C. Hirsch, Cem. Concr. Res. 37 (2007) 537.

[8] F.S. Ortega, V.C. Pandolfelli, J.A. Rodrigues, D.P.F. de Souza, Cerâmica 43 (1997) 113.

[9] D. Gouvêa, B.H. Viana, J. Miagava, Cerâmica 62 (2016) 400.

[10] D. Al Mahrouqi, J. Vinogradov, M.D. Jackson, Adv. Colloid Interface Sci. 240 (2017) 60.

[11] A. Alroudhan, J. Vinogradov, M.D. Jackson, Colloids Surf. A Physicochem. Eng. Asp. 493 (2016) 83.

[12] N.S. Klein, J. Bachmann, A. Aguado, B. TorallesCarbonari, Cem. Concr. Res. 42 (2012) 1611.

[13] L. Galet, S. Patry, J. Dodds, J. Colloid Interface Sci. 346 (2010) 470.

[14] M. Lazghab, K. Saleh, I. Pezron, P. Guigon, L. Komunjer, Powder Technol. 157 (2005) 79.

[15] M. Thakker, V. Karde, D.O. Shah, P. Shukla, C. Ghoroi, Meas. Sci. Technol. 24 (2013) 125902.

[16] A. Alghunaim, S. Kirdponpattara, B.Z. Newby, Powder Technol. 287 (2016) 201.

[17] L. Hu, P. Dong, G. Zhen, Mater. Lett. 63 (2009) 373.

[18] E.W. Washburn, Phys. Rev. 17 (1921) 273.

[19] J.E. Jaine, M.R. Mucalo, Powder Technol. 276 (2015) 123.

[20] A. Siebold, A. Walliser, M. Nardin, M. Oppliger, J. Schultz, J. Colloid Interface Sci. 186 (1997) 60.

[21] A. Alghunaim, B.Z. Newby, Colloids Surf. Physicochem. Eng. Asp. 492 (2016) 79.

[22] S. Kirdponpattara, M. Phisalaphong, B.Z. Newby, J. Colloid Interface Sci. 397 (2013) 169.

[23] L. Susana, F. Campaci, A.C. Santomaso, Powder Technol. 226 (2012) 68.

[24] T. Dang-Vu, J. Hupka, Physicochem. Probl. Miner. Process. 39 (2005) 47.

[25] J. Schoelkopf, P.A.C. Gane, C.J. Ridgway, G.P. Matthews, Colloids Surf. Physicochem. Eng. Asp. 206 (2002) 445.

[26] J.M. Bell, F.K. Cameron, J. Phys. Chem. 10 (1905) 658.

[27] G.P. Matthews, C.W. Watts, D.S. Powlson, J.C. Price, W.R. Whalley, Eur. J. Soil Sci. 59 (2008) 817.

[28] S. Kirchberg, Y. Abdin, G. Ziegmann, Powder Technol. 207 (2011) 311.

[29] S. Ahadian, M. Mohseni, S. Moradian, Int. J. Adhes. Adhes. 29 (2009) 458.

[30] C. Weber, H. Stanjek, J. Colloid Interface Sci. 387 (2012) 270.

[31] Krüss, "Manual do usuário - DSA1 v1.9-03, Análise de formas de gotas - para DSA100" (2004).

[32] N.B. Vargaftik, B.N. Volkov, L.D. Voljak, J. Phys. Chem. Ref. Data 12 (1983) 817.

[33] A.E.R. Westman, H.R. Hugill, J. Am. Ceram. Soc. 13 (1930) 767.

[34] A. Depalo, A.C. Santomaso, Colloids Surf. Physicochem. Eng. Asp. 436 (2013) 371.

(Rec.07/02/2017, Rev. 14/07/2017, Ac. 05/09/2017) 